\title{
ANALYTICAL SOLUTION OF EXCITED TORSIONAL VIBRATIONS OF PRISMATIC THIN-WALLED BEAMS
}

\author{
ElżBieta Augustyn, Marek S. Kozień \\ Cracow University of Technology, Institute of Applied Mechanics, Kraków, Poland \\ e-mail:kozien@mech.pk.edu.pl
}

\begin{abstract}
In the paper, the analytical solution of excited torsional vibrations of prismatic thin-walled beams for different types of boundary conditions and different types of external excitation of torsional moment are formulated. The presented solution can be applied, among others, to preliminary analysis of the optimal position of the actuators and the value of the applied voltage to the elements for minimization of the vibrations of the beams.
\end{abstract}

Keywords: thin-walled beams, torsional vibrations, excited vibrations

\section{Introduction}

Knowledge of the analytical solution of externally excited vibrations for a considered model of realistic structures is very useful for preliminary analysis in the design process or choosing parameters and algorithms for vibration control of the systems. The model of an excited prismatic beam response for torsional or coupled bending-torsional vibrations can be applied for analysis of vibration of some machine elements, e.g. turbine-blades (Gryboś, 1996; Łączkowski, 1974; Pust and Pesek, 2014; Rao, 1991). Sometimes, the beam cross-section takes form of an open thin-walled structure. In this paper, thin-walled beams of closed cross-sections as general models of blades are analyzed (Librescu and Na, 1998; Song and Librescu, 1993; Song et al., 2002).

Recently, application of piezoelectric elements for vibration reduction of thin structures are commonly discussed in the literature (Elliott and Nelson, 1997; Hansen and Snyder, 1997; Moheimani and Fleming, 2006; Preumont, 2006). See also the papers by Ferdek and Kozień (2013), Kozien and Kołtowski (2011). These types of vibration cancellation were considered by the authors in preliminary analysis of the possibility of reduction of torsional vibrations of prismatic beams with solid cross-sections (Augustyn and Kozien, 2014).

There are well known analytical solutions for problems of the dynamics of thin walled beams with open cross-section, starting with Gere (1954), Gere with Lin (1958), Aggrawal with Cranch (1967), and Carr (1969) and later e.g. by Bishop et al. (1989), Dokumaci (1987), Kaliski and Solarz (1992), Tao (1964), Timoshenko et al. (1974) and Yaman (1997). Different cases of vibrations in such a type of structures are discussed in many of articles. A review of these different approaches, theories and models for static and dynamic cases is given by Sapountzakis (2013). The effect of variable cross-section on natural frequencies is discussed in (Eisenberger, 1997). Non-linear models are discussed by Crespo Da Silva (1988a,b), Rozmarynowski with Szymczak (1984) and Di Egidio et al. (2003a,b). The steady-state forced vibrations are discussed by Crespo Da Silva (1988b) and Di Egidio et al. (2003b). The problem of optimal design of a thin-walled beam for a given natural frequency is analyzed by Szymczak (1984). Torsional vibrations of composite thin-walled beams are analyzed by Song and Librescu (1993). The models with the effect of rotary inertia are analysed by Arpaci et al. (2003) and with adaptive capabilities by Song et al. (2002). A detailed solution for the general case of excited vibrations, especially in the 
transient case, are not easily found in the literature. This formulation was given by one of the authors of this paper for bending vibrations of a beam for different types of external excitations (Kozień, 2013).

The main aim of this paper is to formulate the analytical solution for describing the excited torsional-type vibrations of a thin-walled beam for different combinations of boundary conditions (simply supported, free, fixed) and different types of external torsional moment type excitations (harmonic concentrated, harmonic distributed, pulse concentrated, pulse distributed). The presented solution, among others, can be applied to preliminary analysis of the optimal position of the actuators and to calculations of voltage applied to the elements for minimization of vibrations of beams when the application of piezoelectric elements are considered. In this model, the influence of external piezoelectric elements can be modeled by the external concentrated moment of a suitable value, as it was done by Elliott and Nelson (1997), Hansen and Snyder (1997) for pairs of elements in simulations of bending vibrations of beams, as was proposed by Augustyn and Kozień (2014) for double pairs of elements for torsional vibrations of beams.

\section{Formulation of the problem}

\subsection{Equation of motion}

Let us consider a prismatic beam with a thin-walled cross section of an open type (Murray, 1986; Piechnik, 2007; Vlasov, 1959) in which it is also assumed that the material is isotropic. The geometry of the cross-section is shown in Fig. 1, where $G$ is the gravity center (centroid), $S$ - shear center. The origin of the co-ordinate system $S x y z$ lies in the shear center, and the axes $S y$ and $S z$ are parallel to the principal axes of the cross-section $(G \eta, G \zeta)$.

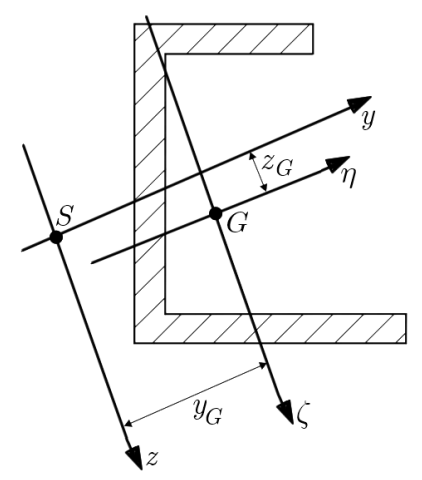

Fig. 1. Geometry of the cross-section

The equations of coupled vibrations of the beam take the following form

$$
\begin{aligned}
& E J_{\zeta} \frac{\partial^{4} v(x, t)}{\partial x^{4}}+\rho A\left[\frac{\partial^{2} v(x, t)}{\partial t^{2}}-z_{G} \frac{\partial^{2} \varphi(x, t)}{\partial t^{2}}\right]=q_{y}(x, t) \\
& E J_{\eta} \frac{\partial^{4} w(x, t)}{\partial x^{4}}+\rho A\left[\frac{\partial^{2} w(x, t)}{\partial t^{2}}+y_{G} \frac{\partial^{2} \varphi(x, t)}{\partial t^{2}}\right]=q_{z}(x, t) \\
& E J_{\omega} \frac{\partial^{4} \varphi(x, t)}{\partial x^{4}}-G J_{s} \frac{\partial^{2} \varphi(x, t)}{\partial x^{2}}+\rho J_{0} \frac{\partial^{2} \varphi(x, t)}{\partial t^{2}} \\
& \quad+\rho A\left[-z_{G} \frac{\partial^{2} v(x, t)}{\partial t^{2}}+y_{G} \frac{\partial^{2} w(x, t)}{\partial t^{2}}\right]=m_{s}(x, t)
\end{aligned}
$$

where $v(x, t)$ is the displacement of the beam in the $y$ direction, $w(x, t)$ is the displacement of the beam in the $z$ direction, $\varphi(x, t)$ is the rotation of the axis of beam around the $x$ direction, $J_{\eta}$ is the principal moment of inertia of the cross-section with respect to the $\eta$ axis, $J_{\zeta}$ is the principal 
moment of inertia of the cross-section with respect to the $\zeta$ axis, $J_{0}$ is the polar moment of inertia with respect to the shear center, $J_{s}$ is the equivalent moment of inertia of the cross-section due to torsion, $E$ is Young's modulus, $G$ is the shear modulus, $\rho$ is the material density, $y_{G}, z_{G}$ are the positions of the gravity center $G$ (co-ordinates), $q_{y}(x, t)$ is the distributed force acting in the $y$ direction (external excitation), $q_{z}(x, t)$ is the distributed force acting in the $z$ direction (external excitation), $m_{s}(x, t)$ is the distributed twisting moment (external excitation).

When the considered cross-section is such that the position of the shear center and gravity center is the same (e.g. for the cross-section with two axes of symetry), the following relationship is valid $y_{G}=z_{G}=0$. It means, that the equation of motion for twisting existing in system (2.1) is separated from the equations of bending vibrations, and takes simplified and independent form from bending vibrations form

$$
\frac{E J_{\omega}}{G J_{s}} \frac{\partial^{4} \varphi(x, t)}{\partial x^{4}}-\frac{\partial^{2} \varphi(x, t)}{\partial x^{2}}+\frac{\rho J_{0}}{G J_{s}} \frac{\partial^{2} \varphi(x, t)}{\partial t^{2}}=\frac{1}{G J_{s}} m_{s}(x, t)
$$

Hence, for the considered cross-section, it is possible to analyze torsional vibrations separately with the bending ones.

If the cross-section is a monolithic type, equation of motion (2.3) takes the following form

$$
-G J_{s} \frac{\partial^{2} \varphi(x, t)}{\partial x^{2}}+\rho J_{0} \frac{\partial^{2} \varphi(x, t)}{\partial t^{2}}=m_{s}(x, t)
$$

In general, vibrations of the analyzed structure can be described in form of a threedimensional model of a solid structure. But for some structures, for which one characteristic dimension (the so-called length) is high enough in comparison to the characteristic dimensions of the cross-section, then so-called one-dimensional models of structures are built for simplicity of the analysis. The results of their application must give good enough results of analysis in comparison with three-dimensional models. The same case takes place during detailed consideration of the cross-section shape. If one characteristic dimension of the cross-section (the so-called thickness) is small enough in comparison with the second one (the so-called width), the whole structure is called a thin-walled one. A detailed rule states that for a thin-walled cross-section, the thickness is more than eight times smaller than the highest way measured along the middle-line of the cross-section between its two end-points. Moreover, the length of this way should be more than eight times smaller than the length of the beam (Piechnik, 2007). For such a beam the assumptions of the Bernoulli-Euler beam theory or the de Saint-Venant rule of loadings, for example, are invalid. Their motion must be analyzed as a three-dimensional body or by the application of an especially formulated theory for thin-walled beams. Due to the form of the middle line of the cross-section, three types of thin-walled beams are defined: with an open cross-section, with a closed cross-section and with a mixed one (Piechnik, 2007). Depending on the type of cross-section, the suitable theory of thin-walled beams should be applied. For a thin-walled beam with an open cross-section, as considered in this article, the Vlasov theory can be applied (Piechnik, 2007; Vlasov, 1959).

\subsection{Boundary conditions}

The equation of motion of the thin-walled beam is the fourth order due to the spatial variable $x$. Therefore, a set of the four boundary conditions must be formulated for each beam element. Their formulation is connected with the Vlasov theory of thin-walled beams and must take into account the warping effect.

The following types of boundary conditions can be formulated in a natural way (Gere, 1954; Piechnik, 2007): 
- Simply supported:

- no rotation of the cross-section around the axis of the beam

$$
\varphi=0
$$

- zero normal stress (free of warping of the cross-section)

$$
\frac{\partial^{2} \varphi}{\partial x^{2}}=0
$$

- Fixed:

- no rotation of the cross-section around the axis of the beam

$$
\varphi=0
$$

- plain cross-section (blocked warping of the cross-section)

$$
\frac{\partial \varphi}{\partial x}=0
$$

- Free:

- zeroes for the total torsional moment (free for rotation)

$$
G J_{s} \frac{\partial \varphi}{\partial x}-E J_{\omega} \frac{\partial \varphi^{3}}{\partial x^{3}}=0
$$

- zero normal stress (free for warping of the cross-section)

$$
\frac{\partial^{2} \varphi}{\partial x^{2}}=0
$$

\section{Eigen-problem, natural vibrations}

Now, the eigen-problem analysis of the thin-walled prismatic beam with length $l$ is considered. The equation of motion has the form

$$
\frac{E J_{\omega}}{G J_{s}} \frac{\partial^{4} \varphi(x, t)}{\partial x^{4}}-\frac{\partial^{2} \varphi(x, t)}{\partial x^{2}}+\frac{\rho J_{0}}{G J_{s}} \frac{\partial^{2} \varphi(x, t)}{\partial t^{2}}=0
$$

The solution to the problem is proposed in form $(3.2)_{1}$ - the Fourier method of solution. After suitable manipulations, it leads to a solution to the problem of separated variables $(3.2)_{2}$. It can be written as two independent ordinary differential equations $(3.2)_{3,4}$. The first of the fourth order for the independent spatial variable $x$, and the second of the second order for the independent variable $t$ (time). The form of unknown functions in the spatial domain can be written in form $\left.(3.2)_{5}\right)$. For the above given homogeneous boundary conditions, these series of functions satisfy orthogonality conditions (3.4) - see Appendix A for detailed analysis. Finally, the solution can be written in form (3.7), where the series of constants $a_{n}$ and $b_{n}$ are determined based on initial conditions (3.6)

$$
\begin{aligned}
& \varphi(x, t)=\sum_{n=1}^{+\infty} X_{n}(x) T_{n}(t) \\
& \frac{E J_{\omega}}{G J_{s}} \frac{\frac{d^{4} X_{n}(x)}{d x^{4}}}{X_{n}(x)}-\frac{\frac{d^{2} X_{n}(x)}{d x^{2}}}{X_{n}(x)}=-\frac{\rho J_{0}}{G J_{s}} \frac{\frac{d^{2} T_{n}(t)}{d t^{2}}}{T_{n}(t)}=\lambda_{n}^{4} \\
& \frac{E J_{\omega}}{G J_{s}} \frac{d^{4} X_{n}(x)}{d x^{4}}-\frac{d^{2} X_{n}(x)}{d x^{2}}-\lambda_{n}^{4} X_{n}(x)=0 \quad \frac{d^{2} T_{n}(t)}{d t^{2}}+\underbrace{\lambda_{n}^{4} \frac{G J_{s}}{\rho J_{0}}}_{\omega_{n}^{2}} T_{n}(t)=0 \\
& X_{n}(x)=A_{n} \sin \left(\alpha_{n} x\right)+B_{n} \cos \left(\alpha_{n} x\right)+C_{n} \sinh \left(\beta_{n} x\right)+D_{n} \cosh \left(\beta_{n} x\right)
\end{aligned}
$$


where

$$
\begin{aligned}
& \alpha_{n}=\sqrt{\frac{-G J_{s}+\sqrt{\left(G J_{s}\right)^{2}+4 E J_{\omega} G J_{s}} \lambda_{n}^{4}}{2 E J_{\omega}}} \\
& \beta_{n}=\sqrt{\frac{G J_{s}+\sqrt{\left(G J_{s}\right)^{2}+4 E J_{\omega} G J_{s}} \lambda_{n}^{4}}{2 E J_{\omega}}} \quad \omega_{n}=\lambda_{n}^{2} \sqrt{\frac{G J_{s}}{\rho J_{0}}}
\end{aligned}
$$

and

$$
\int_{0}^{l} X_{n}(x) X_{m}(x) d x= \begin{cases}\gamma_{n}^{2} & n=m \\ 0 & n \neq m\end{cases}
$$

Finding the solution to the system of differential equation $(3.2)_{2}$ or $(3.2)_{3,4}$ for a given boundary conditions is the well-known eigen-mode problem which gives a set of eigen-values (powered natural frequencies of a system $\omega_{n}^{2}$ - solution of equation $(3.2)_{4}$ and a set of eigenfunctions (eigen-modes, waveforms of eigen-functions $X_{n}(x)$ - solution of equation $\left.(3.2)_{3}\right)$. The equations, which make possible the finding of natural frequencies $\omega_{n}(3.3)_{1,2,3}$ or exact formula for $\omega_{n}$ for a different combination of typical types of boundary conditions (fixed, simply supported, free) are given in Table 1. Moreover, the analytical form of eigen-functions for the same combination of boundary conditions (simply supported, free, fixed) are also given in Table 1. The other name of the problem is the modal problem and the detailed solution of this problem was done by Gere (1954).

If the solution to the equation of motion fulfills initial conditions (3.6), where $\varphi_{0}(x)$ is the initial angle of torsion of the beam for $t=0$, and $\Omega_{0}(x)$ is the initial angular velocity of the beam for $t=0$, the problem of natural vibrations is completely defined. These initial conditions should be applied for the detailed solution of the second equation of system $(3.2)_{3,4}$

$$
\varphi(x, 0)=\varphi_{0}(x) \quad \frac{\partial}{\partial t} \varphi(x, 0)=\Omega_{0}(x)
$$

Solution (3.6) represents the so-called natural vibrations, which are the response of the system to initial conditions written in the time and spatial domains $\varphi(x, t)$

$$
\begin{aligned}
& \varphi(x, t)=\sum_{n=1}^{+\infty} X_{n}(x)\left[a_{n} \sin \left(\omega_{n} t\right)+b_{n} \sin \left(\omega_{n} t\right)\right] \\
& a_{n}=\frac{1}{\omega_{n} \gamma_{n}^{2}} \int_{0}^{l} \Omega_{0}(x) X_{n}(x) d x \quad b_{n}=\frac{1}{\gamma_{n}^{2}} \int_{0}^{l} \varphi_{0}(x) X_{n}(x) d x
\end{aligned}
$$

\section{Excited vibrations}

When analyzing the excited vibrations of a realistic beam (with internal damping), the general solution of the homogeneous differential equation is a function relatively fast tending to zero with respect to time due to the internal and external damping.

The complete solution to the problem of excited vibrations, understood as the particular solution to the non-homogeneous equation (2.2), for the excitation function of harmonic type, has the form of a sum of two components: connected with a set of natural frequencies $\omega_{n}$ and connected with the external loading frequency $\nu$. Note, that the set of constants $A_{n}, B_{n}, C_{n}$ and $D_{n}$ existing in particular solution $(2.2)_{5}$, must be found taking into account the complete solution. 


\begin{tabular}{|c|c|c|}
\hline $\begin{array}{l}\text { Boundary } \\
\text { conditions }\end{array}$ & $\begin{array}{l}\text { Equation/formula for natural } \\
\text { frequency } \omega_{n} \text { or } \\
\text { parameters } \alpha_{n} \text { and } \beta_{n}\end{array}$ & Eigenfunction $X_{n}(x)$ \\
\hline $\begin{array}{l}\text { Simply supported- } \\
\text {-simply supported }\end{array}$ & $\omega_{n}=\frac{n \pi}{l^{2}} \sqrt{\frac{n^{2} \pi^{2} E J_{\omega}+l^{2} G J_{s}}{\rho J_{0}}}$ & $\sin \left(\frac{n \pi}{l} x\right)$ \\
\hline Fixed-fixed & $\alpha_{n} \beta_{n}=\omega_{n} \sqrt{\frac{\rho J_{0}}{E J_{\omega}}}$ & $\begin{aligned} & \frac{\beta_{n}\left[\cosh \left(\beta_{n} l\right)-\cos \left(\alpha_{n} l\right)\right]}{\alpha_{n} \sinh \left(\beta_{n} l\right)-\beta_{n} \sin \left(\alpha_{n} l\right)} \sin \left(\alpha_{n} x\right)-\cos \left(\alpha_{n} x\right) \\
- & \frac{\alpha_{n}\left[\cosh \left(\beta_{n} l\right)-\cos \left(\alpha_{n} l\right)\right]}{\alpha_{n} \sinh \left(\beta_{n} l\right)-\beta_{n} \sin \left(\alpha_{n} l\right)} \sinh \left(\beta_{n} x\right)+\cosh \left(\beta_{n} x\right)\end{aligned}$ \\
\hline $\begin{array}{l}\text { Simply supported- } \\
\text {-fixed }\end{array}$ & $\frac{\tanh \left(\beta_{n} l\right)}{\beta_{n}}=\frac{\tan \left(\alpha_{n} l\right)}{\alpha_{n}}$ & $\sin \left(\alpha_{n} x\right)-\frac{\sin \left(\alpha_{n} l\right)}{\sinh \left(\beta_{n} l\right)} \sinh \left(\beta_{n} x\right)$ \\
\hline Fixed-free & $\begin{array}{c}\frac{\alpha_{n}^{4}+\beta_{n}^{4}}{\alpha_{n}^{2} \beta_{n}^{4}} \cos \left(\alpha_{n} l\right) \cosh \left(\beta_{n} l\right) \\
-\frac{\alpha_{n}^{2}-\beta_{n}^{2}}{\alpha_{n} \beta_{n}} \sin \left(\alpha_{n} l\right) \sinh \left(\beta_{n} l\right)+2=0\end{array}$ & $\begin{array}{l}\frac{\beta_{n}}{\alpha_{n}} \frac{\alpha_{n}^{2} \sinh \left(\beta_{n} l\right)-\alpha_{n} \beta_{n} \sin \left(\alpha_{n} l\right)}{\alpha_{n}^{2} \cosh \left(\beta_{n} l\right)+\beta_{n}^{2} \cos \left(\alpha_{n} l\right)} \sin \left(\alpha_{n} x\right)-\cos \left(\alpha_{n} x\right) \\
-\frac{\alpha_{n}^{2} \sinh \left(\beta_{n} l\right)-\alpha_{n} \beta_{n} \sin \left(\alpha_{n} l\right)}{\alpha_{n}^{2} \cosh \left(\beta_{n} l\right)+\beta_{n}^{2} \cos \left(\alpha_{n} l\right)} \sinh \left(\beta_{n} x\right)+\cosh \left(\beta_{n} x\right)\end{array}$ \\
\hline $\begin{array}{l}\text { Simply supported- } \\
\text {-free }\end{array}$ & $\beta_{n}^{2} \tanh \left(\beta_{n} l\right)=\alpha_{n}^{2} \tan \left(\alpha_{n} l\right)$ & $\sin \left(\alpha_{n} x\right)+\frac{\beta_{n}}{\alpha_{n}} \frac{\cos \left(\alpha_{n} l\right)}{\cosh \left(\beta_{n} l\right)} \sinh \left(\beta_{n} x\right)$ \\
\hline Free-free & $\begin{array}{c}\left(\beta_{n}^{4}-\alpha_{n}^{4}\right) \sin \left(\alpha_{n} l\right) \sinh \left(\beta_{n} l\right) \\
+2 \alpha_{n}^{2} \beta_{n}^{2}\left[\cos \left(\alpha_{n} l\right) \cosh \left(\beta_{n} l\right)-1\right]=0\end{array}$ & $\begin{array}{c}-\frac{\alpha_{n}}{\beta_{n}} \frac{\beta_{n}^{3}\left[\cosh \left(\beta_{n} l\right)-\cos \left(\alpha_{n} l\right)\right]}{\beta_{n}^{3} \sinh \left(\beta_{n} l\right)-\alpha_{n}^{3} \sin \left(\alpha_{n} l\right)} \sin \left(\alpha_{n} x\right)+\frac{\beta_{n}}{\alpha_{n}} \cos \left(\alpha_{n} x\right) \\
-\frac{\beta_{n}^{3}\left[\cosh \left(\beta_{n} l\right)-\cos \left(\alpha_{n} l\right)\right]}{\beta_{n}^{3} \sinh \left(\beta_{n} l\right)-\alpha_{n}^{3} \sin \left(\alpha_{n} l\right)} \sinh \left(\beta_{n} x\right)+\cosh \left(\beta_{n} x\right)\end{array}$ \\
\hline
\end{tabular}




$$
\varphi(x, t)=\sum_{n=1}^{+\infty} X_{n}(x) H_{n}(t)
$$

Putting proposed solution (4.1) into equation (2.2) and taking into account the first of equation of system $(3.2)_{3,4}$ one can obtain

$$
\sum_{n=1}^{+\infty}\left[(\underbrace{\frac{E J_{\omega}}{G J_{s}} \frac{d^{4} X_{n}(x)}{d x^{4}}-\frac{d^{2} X_{n}(x)}{d x^{2}}}_{\lambda_{n}^{4} X_{n}(x)}) H_{n}(t)+\frac{\rho J_{0}}{G J_{s}} \frac{d^{2} H_{n}(t)}{d t^{2}} X_{n}(x)\right]=\frac{1}{G J_{s}} m_{s}(x, t)
$$

and then after suitable manipulations

$$
\sum_{n=1}^{+\infty}[\frac{d^{2} H_{n}(t)}{d t^{2}}+\underbrace{\frac{G J_{s}}{\rho J_{0}} \lambda_{n}^{4}}_{\omega_{n}^{2}} H_{n}(t)] X_{n}(x)=\frac{G J_{s}}{\rho J_{0}} \frac{1}{G J_{s}} m_{s}(x, t)=\frac{1}{\rho J_{0}} m_{s}(x, t)
$$

The external load function existing in equation (2.2) can be represented in a series form (4.4), in terms of functions $X_{n}(x)$ and a series of the known functions of time $Q_{n}(t)$

$$
\begin{array}{ll}
\frac{1}{\rho J_{0}} m_{s}(x, t)=\frac{1}{\rho J_{0}} \sum_{n=1}^{+\infty} X_{n}(x) Q_{n}(t) & \\
Q_{n}(t)=\frac{1}{\gamma_{n}^{2}} \int_{0}^{l} m_{s}(x, t) X_{n}(x) d x & Q_{n}^{*}(t)=\frac{1}{\rho J_{0}} Q_{n}(t)
\end{array}
$$

Substituting formulas (4.1) and (4.4) into equation (2.2), the differential equation for the determination of the unknown functions $H_{n}(t)$ takes the form

$$
\frac{d^{2} H_{n}(t)}{d t^{2}}+\omega_{n}^{2} H_{n}(t)=Q_{n}^{*}(t)
$$

The solution to this equation has the form

$$
H_{n}(t)=\frac{1}{\omega_{n}} \int_{0}^{t} Q_{n}^{*}(\tau) \sin \left[\omega_{n}(t-\tau)\right] d \tau
$$

known as the Duhamel integral.

The given formulas make possible the formulation of the analytical solution of excited vibrations of a beam with defined boundary conditions.

Let us formulate the general form of the Duhamel integral for the following types of external excitations and different boundary conditions given by the eigen-functions $X_{n}(x)$ :

- Harmonically distributed moment with a constant amplitude $m_{0}$

$$
\begin{aligned}
& m_{s}(x, t)=m_{0} \sin (\nu t) \quad\left[m_{0}\right]=N \\
& H_{n}(t)=m_{0} \frac{1}{\rho J_{0}} \frac{1}{\gamma_{n}^{2}} \frac{1}{\omega_{n}^{2}-\nu^{2}}\left[\sin (\nu t)-\frac{\nu}{\omega_{n}} \sin \left(\omega_{n} t\right)\right] \int_{0}^{l} X_{n}(x) d x \\
& \varphi(x, t)=M_{0} \frac{1}{\rho J_{0}} \sum_{n=1}^{\infty} \frac{1}{\gamma_{n}^{2}} \frac{1}{\omega_{n}^{2}-\nu^{2}}\left[\sin (\nu t)-\frac{\nu}{\omega_{n}} \sin \left(\omega_{n} t\right)\right] \int_{0}^{l} X_{n}(x) X_{n}(x) d x
\end{aligned}
$$


- Pulsed distributed moment with a constant amplitude $Q_{m}$ acting at the time $t=t_{0}$

$$
\begin{aligned}
& m_{s}(x, t)=Q_{m} \delta\left(t-t_{0}\right) \quad\left[Q_{m}\right]=N s \\
& H_{n}(t)= \begin{cases}0 & t<t_{0} \\
Q_{M} \frac{1}{\rho J_{0}} \frac{1}{\gamma_{n}^{2}} \frac{1}{\omega_{n}} \sin \left[\omega_{n}\left(t-t_{0}\right)\right] \int_{0}^{l} X_{n}(x) d x & t \geqslant t_{0}\end{cases} \\
& \varphi(x, t)= \begin{cases}0 & t<t_{0} \\
Q_{M} \frac{1}{\rho J_{0}} \sum_{n=1}^{\infty} \frac{1}{\gamma_{n}^{2}} \frac{1}{\omega_{n}} \sin \left[\omega_{n}\left(t-t_{0}\right)\right] \int_{0}^{l} X_{n}(x) X_{n}(x) d x & t \geqslant t_{0}\end{cases}
\end{aligned}
$$

- Harmonic concentrated moment with a constant amplitude $M_{0}$ applied to the point $x=x_{0}$

$$
\begin{aligned}
& m_{s}(x, t)=M_{0} \delta(x-c) \sin (\nu t) \quad\left[M_{0}\right]=N m \\
& H_{n}(t)=M_{0} \frac{1}{\rho J_{0}} \frac{1}{\gamma_{n}^{2}} \frac{1}{\omega_{n}^{2}-\nu^{2}}\left[\sin (\nu t)-\frac{\nu}{\omega_{n}} \sin \left(\omega_{n} t\right)\right] X_{n}\left(x_{0}\right) \\
& \varphi(x, t)=M_{0} \frac{1}{\rho J_{0}} \sum_{n=1}^{\infty} \frac{1}{\gamma_{n}^{2}} \frac{1}{\omega_{n}^{2}-\nu^{2}}\left[\sin (\nu t)-\frac{\nu}{\omega_{n}} \sin \left(\omega_{n} t\right)\right] X_{n}\left(x_{0}\right) X_{n}(x)
\end{aligned}
$$

- Pulsed concentrated moment with a constant amplitude $Q_{m}$ acting at the time $t=t_{0}$ at the point $x=x_{0}$

$$
\begin{aligned}
& m_{s}(x, t)=Q_{M} \delta\left(x-x_{0}\right) \delta\left(t-t_{0}\right) \quad\left[Q_{M}\right]=N m s \\
& H_{n}(t)= \begin{cases}0 & t<t_{0} \\
Q_{M} \frac{1}{\rho J_{0}} \frac{1}{\gamma_{n}^{2}} \frac{1}{\omega_{n}} \sin \left[\omega_{n}\left(t-t_{0}\right)\right] X_{n}\left(x_{0}\right) & t \geqslant t_{0}\end{cases} \\
& \varphi(x, t)= \begin{cases}0 & t<t_{0} \\
Q_{M} \frac{1}{\rho J_{0}} \sum_{n=1}^{\infty} \frac{1}{\gamma_{n}^{2}} \frac{1}{\omega_{n}} \sin \left[\omega_{n}\left(t-t_{0}\right)\right] X_{n}\left(x_{0}\right) X_{n}(x) & t \geqslant t_{0}\end{cases}
\end{aligned}
$$

\section{Example - excited torsional vibrations of a simply supported thin-walled beam}

\subsection{Formulation of the problem}

As an example, let us consider excited vibrations of a thin-walled beam simply supported on both ends. The beam is made of steel $\left(E=2.1 \cdot 10^{11} \mathrm{~Pa}, G=8.1 \cdot 10^{10} \mathrm{~Pa}, \rho=7800 \mathrm{~kg} / \mathrm{m}^{3}\right)$ and has length $l=6 \mathrm{~m}$. The shape of cross-section with its detailed dimensions is shown in Fig. 2 .

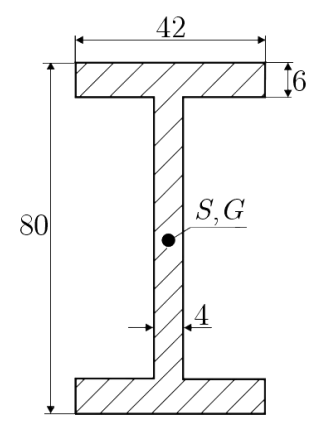

Fig. 2. Cross-section of the analyzed beam (dimensions given in $\mathrm{mm}$ ) 
The boundary conditions take form

$$
\begin{aligned}
\varphi(0, t) & =0 & \frac{\partial^{2}}{\partial x^{2}} \varphi(0, t) & =0 \\
\varphi(l, t) & =0 & \frac{\partial^{2}}{\partial x^{2}} \varphi(l, t) & =0
\end{aligned}
$$

\subsection{Natural vibrations - a comparison of the results for different models}

The following models of beam are applied for comparison of the lowest natural frequencies for torsional vibrations:

- three-dimensional solid model solved by application of the finite element method,

- thin-walled Vlasov analytical model,

- model with a monolithic cross-section.

The finite element solution has been obtained by application of the Ansys finite element package. The model is built of 76800 three dimensional 8-nodes solid elements of the type solid45. The generated mesh is shown in Fig. 3. Due to the degrees of freedom of the applied elements, the boundary conditions on the two ends of the beam are modeled as free sliding along the axis of the beam and as blocked displacements for all nodes in the plane of its ends. Therefore, deplanation of the ends is possible.

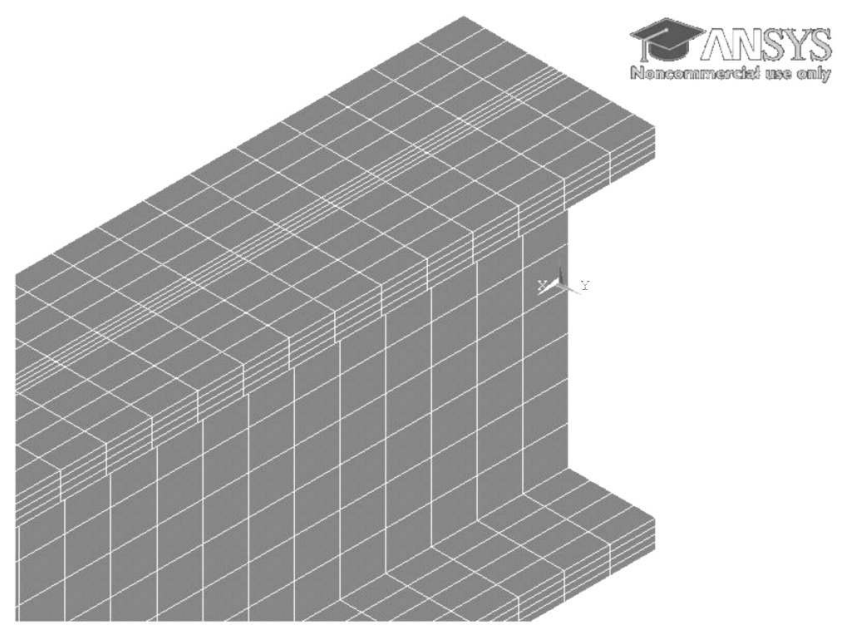

Fig. 3. Part of the FEM mesh of the beam

The second group of values of the natural frequencies is obtained based on the thin-walled model described in the text, especially based on the formula given in the first row in Table 1. The values of $J_{s}, J_{\omega}$ and $J_{0}$ are calculated based on the formulas given by Gere (1954).

The third group of results is obtained for the beam modeled with the assumption of a monolithic type cross-section (shaft) with free-free $(\varphi(0, t)=\varphi(l, t)=0)$ or fixed-fixed $(\partial \varphi(0, t) / \partial x=\partial \varphi(l, t) / \partial x=0)$ boundary conditions. The values of natural frequencies are the same for these cases of boundary conditions (Woroszył, 1984).

In Table 2, a comparison of the lowest natural frequencies for the analyzed beam for the three considered models and the relative percentage error is given to one model obtained from three dimensional solid models, as the reference model. It should be noted that for the considered beam, the values of natural frequencies are correctly estimated theoretically with a monolithic cross-section only for the first few torsional modes. For higher modes, only the theory for thinwalled beam gives good enough results. 
Table 2. Comparison of the lowest natural frequencies for the analyzed beam $[\mathrm{Hz}]$

\begin{tabular}{|c|c|c|c|c|c|}
\hline Mode & 3-D Solid & \multicolumn{2}{|c|}{ Thin-walled } & \multicolumn{2}{c|}{ Monolytic } \\
\hline No. & value [Hz] & value [Hz] & error [\%] & value [Hz] & error [\%] \\
\hline \hline 1 & 25.15 & 25.54 & 1.6 & 25.40 & 1.0 \\
\hline 2 & 50.97 & 51.89 & 1.8 & 50.81 & -0.3 \\
\hline 3 & 78.11 & 79.84 & 2.2 & 76.21 & -2.4 \\
\hline 4 & 107.16 & 110.05 & 2.7 & 101.62 & -5.2 \\
\hline 5 & 138.64 & 143.16 & 3.3 & 127.02 & -8.4 \\
\hline 6 & 172.98 & 179.66 & 3.9 & 152.42 & -11.9 \\
\hline 7 & 210.56 & 219.94 & 4.5 & 177.83 & -15.5 \\
\hline 8 & 251.65 & 264.36 & 5.1 & 203.23 & -19.2 \\
\hline 9 & 296.50 & 313.14 & 5.6 & 228.64 & -22.9 \\
\hline 10 & 345.26 & 366.49 & 6.1 & 254.04 & -26.4 \\
\hline
\end{tabular}

A comparison of the natural frequencies for the two beam models - the thin-walled one and the monolithic one are shown in Fig. 4 in form of the relative percentage error in comparison to the values obtained for the thin-walled model as the reference model. On the horizontal axis, there are identification numbers of the first hundred torsional modes $(n=1,2, \ldots, 100)$. It can be shown that for the considered beam, the error of the estimated values of natural frequencies found by application of the theory with monolithic cross-section grow rapidly with an increase in the number of torsional modes.

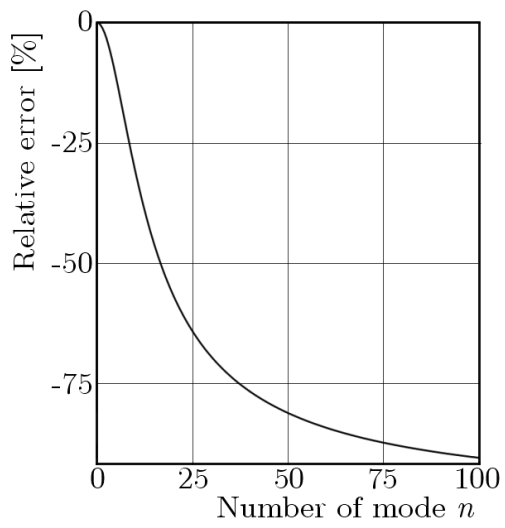

Fig. 4. Relative error of the natural frequencies for torsional modes for the beam models

\subsection{Excited vibrations}

The vibrations are excited by a concentrated moment with an amplitude $M_{0}=5 \mathrm{Nm}$ acting at the point distanced $0.4 l(2.4 \mathrm{~m})$ from the end of beam.

The initial conditions are zeroes (i.e $\varphi_{0}(x)=0$ and $\Omega_{0}=0$ in formulas (3.6))

$$
m_{s}(x, t)=M_{0} \delta(x-0.4 l) \sin (\nu t)
$$

The angular frequency of the excitation is $\nu=200 \mathrm{rad} / \mathrm{s}$. Material damping is neglected. The analytical solution has the form

$$
\varphi(x, t)=\frac{2}{l} \frac{1}{\rho J_{0}} M_{0} \sum_{n=1}^{\infty} \frac{1}{\omega_{n}^{2}-\nu^{2}}\left[\sin (\nu t)-\frac{\nu}{\omega_{n}} \sin \left(\omega_{n} t\right)\right] \sin \left(\lambda_{n} 0.4 l\right) \sin \left(\lambda_{n} x\right)
$$

In the analysis, the first five modes are considered. 
The displacement of the middle of the beam just after the beginning of vibrations and for the steady-state are shown in Fig. 5. The control point is the place of action of the concentrated excitation moment. It is distanced by $0.4 l(2.4 \mathrm{~m})$ from the end of the beam.

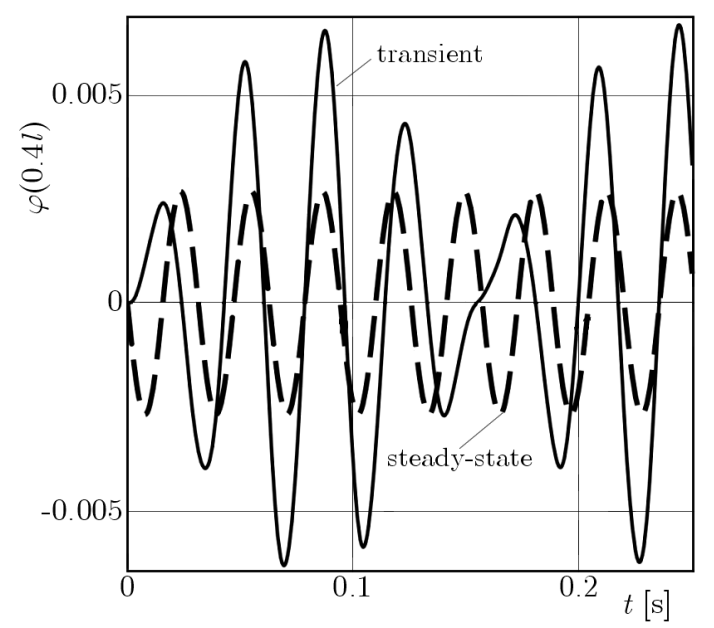

Fig. 5. Displacement at the control point - complete analytical solution, transient and steady-state

The plots show the necessity of taking into account the full solution instead of the steadystate case if the results are important just after the start of action of external loadings. Due to internal material damping (not taken into account in the considered model), the transient component existing in solution is a function that relatively fastly tends to zero. Therefore, enough time after the start of action of the harmonic excitation, the solution takes the steady-state form.

\section{Conclusions}

- The solution of the problem for a monolithic cross-section is not an asymptotic case of the solution of the thin-walled case if the value of $J_{\omega}$ tends to zero.

- The given formulas can be applied to the analysis of reduction of torsional vibrations by coupled sets of piezoelectric elements. The action of piezoelectric elements can be approximately modeled by a concentrated moment put in the suitable cross-section, see Elliott and Nelson (1997), Hansen and Snyder (1997) for beams.

- The given formulas make it possible to find an analytical solution for the transient case of the response (just after the excitation is applied) and for the steady-state case.

- The solution can be generalized by assuming the Voigt-Kelvin model of the viscous type of damping for description of the internal material damping.

\section{Appendix A. Orthogonality of the eigen-functions}

Formula $(3.2)_{2}$ can be written in the following form

$$
\begin{aligned}
& G J_{s} \lambda_{n}^{4} X_{n}(x)=E J_{\omega} \frac{d^{4} X_{n}(x)}{d x^{4}}-G J_{s} \frac{d^{2} X_{n}(x)}{d x^{2}} \\
& G J_{s} \lambda_{m}^{4} X_{m}(x)=E J_{\omega} \frac{d^{4} X_{m}(x)}{d x^{4}}-G J_{s} \frac{d^{2} X_{m}(x)}{d x^{2}}
\end{aligned}
$$

Let us multiply the first equation of system (A.1) by the eigen-function $X_{m}(x)$ and the second equation suitably by $X_{n}(x)$, and then subtract the equations by sides and integrate. 
Finally, formula (A.1) is

$$
\begin{gathered}
G J_{s}\left(\lambda_{n}^{4}-\lambda_{m}^{4}\right) \int_{0}^{l} X_{n}(x) X_{m}(x) d x=E J_{\omega} \int_{0}^{l} \frac{d^{4} X_{n}(x)}{d x^{4}} X_{m}(x) d x-E J_{\omega} \int_{0}^{l} \frac{d^{4} X_{m}(x)}{d x^{4}} X_{n}(x) d x \\
-G J_{s} \int_{0}^{l} \frac{d^{2} X_{n}(x)}{d x^{2}} X_{m}(x) d x+G J_{s} \int_{0}^{l} \frac{d^{2} X_{m}(x)}{d x^{2}} X_{n}(x) d x
\end{gathered}
$$

By integrating the right side of equation (4.2) by parts, one obtains the following formulas for each component

$$
\begin{aligned}
& E J_{\omega} \int_{0}^{l} \frac{d^{4} X_{n}(x)}{d x^{4}} X_{m}(x) d x \\
& \quad=E J_{\omega}\left[\left.\frac{d^{3} X_{n}(x)}{d x^{3}} X_{m}(x)\right|_{0} ^{l}-\left.\frac{d^{2} X_{n}(x)}{d x^{2}} \frac{d X_{m}(x)}{d x}\right|_{0} ^{l}+\int_{0}^{l} \frac{d^{2} X_{n}(x)}{d x^{2}} \frac{d^{2} X_{m}(x)}{d x^{2}} d x\right] \\
& E J_{\omega} \int_{0}^{l} \frac{d^{4} X_{m}(x)}{d x^{4}} X_{n}(x) d x \\
& \quad=E J_{\omega}\left[\left.\frac{d^{3} X_{m}(x)}{d x^{3}} X_{n}(x)\right|_{0} ^{l}-\left.\frac{d^{2} X_{m}(x)}{d x^{2}} \frac{d X_{n}(x)}{d x}\right|_{0} ^{l}+\int_{0}^{l} \frac{d^{2} X_{m}(x)}{d x^{2}} \frac{d^{2} X_{n}(x)}{d x^{2}} d x\right] \\
& G J_{s} \int_{0}^{l} \frac{d^{2} X_{n}(x)}{d x^{2}} X_{m}(x) d x=\left.G J_{s} \frac{d X_{n}(x)}{d x} X_{m}(x)\right|_{0} ^{l}-\int_{0}^{l} \frac{d X_{n}(x)}{d x} \frac{d X_{m}(x)}{d x} d x \\
& G J_{s} \int_{0}^{l} \frac{d^{2} X_{m}(x)}{d x^{2}} X_{n}(x) d x=\left.G J_{s} \frac{d X_{m}(x)}{d x} X_{n}(x)\right|_{0} ^{l}-\int_{0}^{l} \frac{d X_{m}(x)}{d x} \frac{d X_{n}(x)}{d x} d x
\end{aligned}
$$

Finally, the relationship (A.2) can be written in the form

$$
\begin{aligned}
G J_{s} & \left(\lambda_{n}^{4}-\lambda_{m}^{4}\right) \int_{0}^{l} X_{n}(x) X_{m}(x) d x=\left(E J_{\omega} \frac{d^{3} X_{n}(l)}{d x^{3}}-G J_{s} \frac{d^{2} X_{n}(l)}{d x^{2}}\right) X_{m}(l) \\
& -\left(E J_{\omega} \frac{d^{3} X_{n}(0)}{d x^{3}}-G J_{s} \frac{d^{2} X_{n}(0)}{d x^{2}}\right) X_{m}(0)-\left(E J_{\omega} \frac{d^{3} X_{m}(l)}{d x^{3}}-G J_{s} \frac{d^{2} X_{m}(l)}{d x^{2}}\right) X_{n}(l) \\
& +\left(E J_{\omega} \frac{d^{3} X_{m}(0)}{d x^{3}}-G J_{s} \frac{d^{2} X_{m}(0)}{d x^{2}}\right) X_{n}(0)-E J_{\omega} \frac{d^{2} X_{n}(l)}{d x^{2}} \frac{d X_{m}(l)}{d x} \\
& +E J_{\omega} \frac{d^{2} X_{n}(0)}{d x^{2}} \frac{d X_{m}(0)}{d x} E J_{\omega} \frac{d^{2} X_{m}(l)}{d x^{2}} \frac{d X_{n}(l)}{d x}-E J_{\omega} \frac{d^{2} X_{m}(0)}{d x^{2}} \frac{d X_{n}(0)}{d x}
\end{aligned}
$$

For any physically possible combination of the discussed boundary conditions, all components existing on the right-hand side are equal to zero. It means that for $n \neq m$

$$
\int_{0}^{l} X_{n}(x) X_{m}(x) d x=0
$$

For $n=m$

$$
\int_{0}^{l} X_{n}(x) X_{n}(x) d x=\gamma_{n}^{2}
$$


The value of $\gamma_{n}^{2}$ depends on the form of the eigen-functions (see Table 1).

These relationships are known as the orthogonality conditions of the eigen-function $X_{n}(x)$.

\section{References}

1. Aggarwal H.R., Cranch E.T., 1967, A theory of torsional and coupled bending torsional waves in thin-walled open section beams, Journal of Applied Mechanics - Transactions of the ASME, 34, $6,337-343$

2. Arpaci A., Bozdag S.E., Sunbuloglu E., 2003, Triply coupled vibrations of thin-walled open cross-section beams including rotary inertia effects, Journal of Sound and Vibration, 260, 889-900

3. Augustyn E., Kozień M.S., 2014, Study of possibility of application of piezoelectric actuators for active reduction of torsional vibrations of beams, Acta Physica Polonica A, 125, 4-A, 164-168

4. Bishop R.E.D., Cannon M., Miao S., 1989, On coupled bending and torsional vibration of uniform beams, Journal of Sound and Vibration, 131, 1, 457-464

5. CARr J.B., 1969, Torsional vibration of uniform thin-walled beams of open section, Aeronautical Journal, 73, 704, 672-674

6. Crespo Da Silva M.R.M., 1988a, Non-linear flexural-flexural-torsional-extensional dynamics of beams - I. Formulation, International Journal of Solids and Structures, 24, 12, 1225-1234

7. Crespo Da Silva M.R.M., 1988b, Non-linear flexural-flexural-torsional-extensional dynamics of beams - II. Response analysis, International Journal of Solids and Structures, 24, 12, 1235-1242

8. Di Egidio A., Luongo A., Vestroni F., 2003a, A non-linear model for the dynamics of open cross-section thin-walled beams - Part I: formulation, International Journal of Non-Linear Mechanics, 38, 7, 1067-1081

9. Di Egidio A., Luongo A., Vestroni F., 2003b, A non-linear model for the dynamics of open cross-section thin-walled beams - Part II: forced motion, International Journal of Non-Linear Mechanics, 38, 7, 1083-1094

10. Dokumaci E., 1987, An exact solution for coupled bending and torsion vibrations of uniform beams having single cross-sectional symmetry, Journal of Sound and Vibration, 119, 3, 443-449

11. Eisenberger M., 1997, Torsional vibrations of open and variable cross-section bars, Thin-Walled Structures, 27, 3/4, 269-278

12. Elliott S.P., Nelson P.A., 1997, Active Control of Vibrations, Academic Press, London

13. Ferdek U., Kozień M.S., 2013, FEM simulation of application of FGM piezoelectric actuators for active reduction of beam vibrations, Acta Physica Polonica A, 123, 6, 1044-1047

14. Gere J.M., 1954, Torsional vibrations of beams of thin-walled open section, Journal of Applied Mechanics - Transactions of the ASME, 21, 4, 381-387

15. Gere J.M., Lin Y.K., 1958, Coupled vibrations of thin-walled beams of open cross section, Journal of Applied Mechanics - Transactions of the ASME, 25, 3, 373-378

16. Gryboś R., 1996, Vibrations of Machines (in Polish), Politechnika Sląska, Gliwice

17. Hansen C.H., Snyder S.D., 1997, Active Control of Noise and Vibration, E\&FN Spon, London

18. Kaliski S., Solarz L. (Eds.), 1992, Vibrations and Waves. Part B: Waves, Elsevier

19. Kozień M.S., 2013, Analytical solutions of excited vibrations of a beam with application of distribution, Acta Physica Polonica A, 123, 6, 1029-1033

20. Kozień M.S., Koєtowski B., 2011, Comparison of active and passive damping of plate vibration by piezoelectric actuators - FEM simulation, Acta Physica Polonica A, 119, 6-A, 1005-1008

21. Librescu L., NA S., 1998, Dynamic response control of thin-walled beams to blast pulses using structural tailoring and piezoelectric actuation, Journal of Applied Mechanics, 65, 2, 497-504 
22. Łączkowski R., 1974, Vibrations of Elements of Heat Turbines (in Polish), WNT, Warszawa

23. Moheimani S.O.R., Fleming A.J., 2006, Piezoelectric Transducers for Vibration Control and Damping, Springer, London

24. Murray N.W., 1986, Introduction to the theory of thin-walled structures, Oxford University Press, Oxford

25. Piechnik S., 2007, Technical Mechanics of Solid Body, Politechnika Krakowska, Kraków

26. Pust L., Pesek L., 2014, Blade couple connected by damping element with dry friction, Journal of Theoretical and Applied Mechanics, 52, 3, 815-826

27. Preumont A., 2006, Mechatronics. Dynamics of Electromechanical and Piezoelectric Systems, Springer, Berlin

28. RaO J.S., 1991, Turbomachine Blade Vibration, Wiley, Chichester

29. Rozmarynowski B., Szymczak C., 1984, Non-linear free torsional vibrations of thin-walled beams with bisymmetric cross-section, Journal of Sound and Vibration, 97, 1, 145-152

30. Sapountzaskis E.J., 2013, Bars under torsional loading: A generalized beam theory approach, ISRN Civil Engineering, Article ID 916581, http://dx.doi.org/10.1155/2013/916581

31. Song O., Librescu L., 1993, Free vibrations of anisotropic composite thin-walled beams of closed cross-section contour, Journal of Sound and Vibration, 167, 1, 129-147

32. Song O., Sang-Yong O.H., Librescu L., 2002, Dynamic behavior of elastically tailored rotating blades modeled as pretwisted thin-walled beams and incorporating adaptive capabilities, International Journal of Rotating Machinery

33. SzymczaK C., 1984, Optimal design of thin-walled I beams for a given natural frequency of torsional vibrations, Journal of Sound and Vibration, 97, 1, 137-144

34. TAo W.K., 1964, Dynamics of thin-walled beams of open section, Ph.D. Thesis, California Institute of Technology, Pasadena

35. Timoshenko S.P., Young D.H., Weaver W. JR., 1974, Vibration Problems in Engineering, Wiley, New York

36. Vlasov V.Z., 1959, Thin-Walled Elastic Beams (in Russian), Goz. Izd. Fiz. Mat. Literat., Moskwa

37. WoroszyŁ S., 1984, Examples and Exercises from Theoty of Vibrations (in Polish), PWN, Warszawa

38. YAmAN Y., 1997, Vibration of open-section channels: a coupled flexural and torsional wave analysis, Journal of Sound and Vibration, 204, 1, 131-158 